\title{
PULLBACK ATTRACTORS FOR A NON-AUTONOMOUS SEMI-LINEAR DEGENERATE PARABOLIC EQUATION
}

\author{
CUNG THE ANH and TANG QUOC BAO \\ Department of Mathematics, Hanoi National University of Education, 136 Xuan Thuy, \\ Cau Giay, Hanoi, Vietnam \\ e-mail:anhctmath@hnue.edu.vn,mathizonewww@gmail.com
}

(Received 8 May 2009; revised 28 October 2009; accepted 21 February 2010)

\begin{abstract}
In this paper, using the asymptotic a priori estimate method, we prove the existence of pullback attractors for a non-autonomous semi-linear degenerate parabolic equation in an arbitrary domain, without restriction on the growth order of the polynomial type non-linearity and with a suitable exponential growth of the external force. The obtained results improve some recent ones for the non-autonomous reaction-diffusion equations.
\end{abstract}

2010 Mathematics Subject Classification. 35B41, 35K65, 35D05.

1. Introduction. In this paper, we study the following non-autonomous semilinear degenerate parabolic equation with variable, non-negative coefficients, defined on an arbitrary domain (bounded or unbounded) $\Omega \subset \mathbb{R}^{N}, N \geq 2$,

$$
\begin{aligned}
\frac{\partial u}{\partial t}-\operatorname{div}(\sigma(x) \nabla u)+f(u) & =g(t), \quad x \in \Omega, t>\tau, \\
\left.u\right|_{t=\tau} & =u_{\tau}(x), \quad x \in \Omega, \\
\left.u\right|_{\partial \Omega} & =0,
\end{aligned}
$$

where $u_{\tau} \in L^{2}(\Omega)$ is given, $f \in C(\mathbb{R})$ satisfies

$$
\begin{gathered}
C_{1}|u|^{p}-k_{1} \leq f(u) u \leq C_{2}|u|^{p}+k_{2}, p \geq 2, \\
(f(u)-f(v))(u-v) \geq-l|u-v|^{2},
\end{gathered}
$$

and the external force $g$ satisfies

$$
g \in L_{l o c}^{2}\left(\mathbb{R} ; L^{2}(\Omega)\right) \text {, and }\|g(t)\|_{L^{2}(\Omega)}^{2} \leq M e^{\gamma|t|},
$$

where $C_{1}, C_{2}, k_{1}, k_{2}, l, M$ are positive constants, $\gamma<\lambda_{1}$, where $\lambda_{1}>0$ is the first eigenvalue of the operator $A:=-\operatorname{div}(\sigma(x) \nabla)$ in $\Omega$ with the homogeneous Dirichlet condition (see Section 2.1).

Problem (1.1) can be derived as a simple model for neutron diffusion (feedback control of nuclear reactor) (see [13]). In this case $u$ and $\sigma$ stand for the neutron flux and neutron diffusion respectively.

The degeneracy of problem (1.1) is considered in the sense that the measurable, non-negative diffusion coefficient $\sigma(x)$, is allowed to have at most a finite number 
of (essential) zeroes at some points. Motivated by [8], where a degenerate elliptic problem is studied, we assume that the function $\sigma: \Omega \rightarrow \mathbb{R}$ satisfies the following assumptions:

$\left(\mathcal{H}_{\alpha}\right) \sigma \in L_{\text {loc }}^{1}(\Omega)$ and for some $\alpha \in(0,2), \liminf _{x \rightarrow z}|x-z|^{-\alpha} \sigma(x)>0$ for every $z \in$ $\bar{\Omega}$, when the domain $\Omega$ is bounded.

$\left(\mathcal{H}_{\alpha, \beta}^{\infty}\right) \sigma$ satisfies condition $\left(\mathcal{H}_{\alpha}\right)$ and $\lim \inf |x| \rightarrow \infty|x|^{-\beta} \sigma(x)>0$ for some $\beta>2$, when the domain $\Omega$ is unbounded.

For the physical motivation of the assumptions $\left(\mathcal{H}_{\alpha}\right)$ and $\left(\mathcal{H}_{\alpha, \beta}^{\infty}\right)$, we refer the reader to $[2,8,14,15]$.

In order to study problem (1.1) we use the natural energy space $\mathcal{D}_{0}^{1}(\Omega, \sigma)$ defined as the closure of $C_{0}^{\infty}(\Omega)$ in the norm

$$
\|u\|_{\mathcal{D}_{0}^{1}(\Omega, \sigma)}:=\left(\int_{\Omega} \sigma(x)|\nabla u|^{2} d x\right)^{1 / 2} .
$$

This space is a Hilbert space with respect to the scalar product

$$
(u, v)_{\sigma}:=\int_{\Omega} \sigma(x) \nabla u \nabla v d x
$$

The existence and long-time behaviour of solutions of the problem (1.1) in the autonomous case have been studied in $[\mathbf{1 4}, \mathbf{1 5}]$ and improved recently in [2]. In [2], the authors considered the problem (1.1) with $u_{0} \in \mathcal{D}_{0}^{1}(\Omega, \sigma), g \in L^{2}(\Omega)$ given, and $f: \mathbb{R} \rightarrow \mathbb{R}$ satisfies

$$
\begin{gathered}
|f(u)-f(v)| \leq C_{0}|u-v|\left(1+|u|^{\gamma}+|v|^{\gamma}\right), 0 \leq \gamma<\frac{4-2 \alpha}{N-2+\alpha}, \\
F(u) \geq-\frac{\mu}{2} u^{2}-C_{1}, \\
f(u) u \geq-\mu u^{2}-C_{2},
\end{gathered}
$$

where $C_{0}, C_{1}, C_{2} \geq 0, F$ is the primitive $F(y)=\int_{0}^{y} f(s) d s$ of $f, \mu<\lambda_{1}, \lambda_{1}$ is the first eigenvalue of the operator $A u:=-\operatorname{div}(\sigma(x) \nabla u)$ in $\Omega$ with homogeneous Dirichlet boundary conditions. Under the above assumptions of $f$, the authors proved that problem (1.1) defines a semi-group $S(t): \mathcal{D}_{0}^{1}(\Omega, \sigma) \rightarrow \mathcal{D}_{0}^{1}(\Omega, \sigma)$, which possesses a compact connected global attractor $\mathcal{A}=W^{u}(E)$ in the space $\mathcal{D}_{0}^{1}(\Omega, \sigma)$. Furthermore, for each $u_{0} \in \mathcal{D}_{0}^{1}(\Omega, \sigma)$, the corresponding solution $u(t)$ tends to the set $E$ of equilibrium points in $\mathcal{D}_{0}^{1}(\Omega, \sigma)$ as $t \rightarrow+\infty$. The basic tool for the approach in this case is the following Lyapunov function

$$
\Phi(u)=\frac{1}{2}\|u\|_{\mathcal{D}_{0}^{1}(\Omega, \sigma)}^{2}+\int_{\Omega}(F(u)+g u) d x .
$$

Noting that the critical exponent of the embedding $\mathcal{D}_{0}^{1}(\Omega, \sigma) \hookrightarrow L^{p}(\Omega)$ is $2_{\alpha}^{*}=\frac{2 N}{N-2+\alpha}$, so the condition $0 \leq \gamma<\frac{4-2 \alpha}{N-2+\alpha}$ is necessary to prove the existence of a mild solution by the fixed point method and to ensure the existence of the Lyapunov function $\Phi$.

In this paper, we continue studying the long-time behaviour of solutions to problem (1.1) by removing the restrictions on the growth of the non-linearity $f$ and allowing the external force $g$ depending on time $t$. Non-autonomous equations appear in many 
applications in the natural sciences, so they are also of great importance and interest. The long-time behaviour of solutions of such equations have been studied extensively in the last years. The first attempt was to extend the notion of global attractor to the non-autonomous case leading to the concept of the so-called uniform attractor (see [12]). It is remarkable that the conditions ensuring the existence of the uniform attractor parallel those for autonomous equations. To this end, non-autonomous systems are lifted in [23] to autonomous ones by expanding the phase space. Then, the existence of uniform attractor relies on some compactness properties of the solution operator associated to the system. However, one disadvantage of the uniform attractor is that it need not to be 'invariant' unlike the global attractor for autonomous systems. In order to overcome this drawback, a new concept, called pullback attractor, has been introduced for the non-autonomous case. The theory of pullback attractors has been developed for both the non-autonomous and random dynamical systems and has shown to be very useful in the understanding of the dynamics of non-autonomous dynamical systems (see [9] and references therein).

On the other hand, generalised semi-flows are an abstraction of autonomous dynamical systems for which there may be more than one solution corresponding to given initial data. As mentioned in [6], the need for a theory of such systems arises for various reasons. First, there may be genuine non-uniqueness of solutions. Second, solutions may not be known to be unique (as, for example, for certain semi-linear wave equations with high power non-linearities, or for the incompressible NavierStokes equations in three space dimension). Third, there may be free parameters or controls that are not specified and lead to various possible solutions. For the qualitative analysis of such systems from the point of view of the theory of dynamical systems, it is necessary to develop a corresponding theory for multi-valued semi-groups. In the last years, there have been some theories for which one can treat multi-valued semi-flows and their asymptotic behaviour, such as generalised semi-flows theory of Ball [5-7] and theory of multi-valued semi-flows of Menlik and Valero [21]. A comparision of these two theories can be found in [10]. We note also that the theory of trajectory attractors of Chepyzhov and Vishik has been also fruitfully applied to treat equations without uniqueness $[\mathbf{1 1}, \mathbf{1 2}]$. Thanks to these theories, the asymptotic behaviour of equations without uniqueness of the Cauchy problems has been studied by several authors in the last years (see e.g. $[3,4]$ for the long-time behaviour for quasilinear degenerate equations with weights of the above type).

The main aim of this paper is to prove the existence of a pullback $\mathcal{D}$-attractor in the space $\mathcal{D}_{0}^{1}(\Omega, \sigma) \cap L^{p}(\Omega)$ for the process generated by problem (1.1). Let us describe the methods used in the paper. First, we use the compactness method [11] to prove the global existence of a weak solution and use a priori estimates to show the existence of a family of pullback $\mathcal{D}$-absorbing sets $\hat{B}=\{B(t): t \in \mathbb{R}\}$ in $\mathcal{D}_{0}^{1}(\Omega, \sigma) \cap L^{p}(\Omega)$ ) for the process. By the compactness of the embedding $\mathcal{D}_{0}^{1}(\Omega, \sigma) \hookrightarrow L^{2}(\Omega)$, the process is pullback $\mathcal{D}$-asymptotically compact in $L^{2}(\Omega)$. This immediately implies the existence of a pullback $\mathcal{D}$-attractor in $L^{2}(\Omega)$. When proving the existence of pullback $\mathcal{D}$-attractors in $L^{p}(\Omega)$ and in $\mathcal{D}_{0}^{1}(\Omega, \sigma) \cap L^{p}(\Omega)$, to overcome the difficulty arising by the lack of embedding results, we use the asymptotic a priori estimate method initiated in [20] for autonomous equations and developed in [19] for non-autonomous equations. One of the main new features in our paper is that the existence of a pullback $\mathcal{D}$-attractor is proved for a class of semi-linear degenerate parabolic equations in an arbitrary (bounded or unbounded) domain. It is also worth noticing that, when $\sigma=1$, our 
results improve the recent results in $[\mathbf{1 6}, \mathbf{1 7}, \mathbf{2 2}]$ for the non-autonomous Laplacian equation in bounded domains.

The content of the paper is as follows. In Section 2, for the convenience of the reader, we recall some concepts and results on function spaces and pullback attractors which we will use. For clarity, in Sections 3 and 4, we only consider the case of a bounded domain and the diffusion coefficient $\sigma$ satisfying condition $\left(\mathcal{H}_{\alpha}\right)$. Section 3 is devoted to the proof of the global existence and uniqueness of a weak solution to problem (1.1) by using the compactness method. In Section 4, we prove the existence of pullback attractors in various spaces by using the asymptotic a priori estimate method. In the last section, we give some remarks on similar results for an unbounded domain and $\sigma$ satisfying condition $\left(\mathcal{H}_{\alpha, \beta}^{\infty}\right)$.

\section{Preliminaries.}

2.1. Function spaces and operator. We recall some basic results on the function spaces which we will use. Let $N \geq 2, \alpha \in(0,2)$, and

$$
2_{\alpha}^{*}= \begin{cases}\frac{4}{\alpha} \in(2, \infty) & \text { if } N=2, \\ \frac{2 N}{N-2+\alpha} \in\left(2, \frac{2 N}{N-2}\right) & \text { if } N \geq 3 .\end{cases}
$$

The exponent $2_{\alpha}^{*}$ has the role of the critical exponent in the classical Sobolev embedding. We have the following generalised version of the Poincare inequality ([4, Corollary 2.6]).

Lemma 2.1. Let $\Omega$ be a bounded (unbounded) domain in $\mathbb{R}^{N}, N \geqslant 2$, and assume that condition $\left(\mathcal{H}_{\alpha}\right)\left(\left(\mathcal{H}_{\beta}^{\infty}\right)\right)$ is satisfied. Then there exists a constant $c>0$, such that

$$
\int_{\Omega}|u|^{2} d x \leqslant c \int_{\Omega} \sigma(x)|\nabla u|^{2} d x, \text { for every } u \in C_{0}^{\infty}(\Omega) .
$$

We emphasize that condition $\left(\mathcal{H}_{\alpha}\right)$ is optimal in the following sense: For $\alpha>2$ there exist functions such that (2.1) is not satisfied (see [8]). Note also that in the case of an unbounded domain, (2.1) does not hold in general, if $\beta \leqslant 2$ in $\left(\mathcal{H}_{\alpha, \beta}^{\infty}\right)$. We refer also to the examples of [1].

The natural energy space for problem (1.1) involves the space $\mathcal{D}_{0}^{1}(\Omega, \sigma)$, defined as the closure of $C_{0}^{\infty}(\Omega)$ with respect to the norm

$$
\|u\|_{\mathcal{D}_{0}^{1}(\Omega, \sigma)}:=\left(\int_{\Omega} \sigma(x)|\nabla u|^{2} d x\right)^{\frac{1}{2}} .
$$

The space $\mathcal{D}_{0}^{1}(\Omega, \sigma)$ is a Hilbert space with respect to the scalar product

$$
(u, v)_{\sigma}:=\int_{\Omega} \sigma(x) \nabla u \nabla v d x .
$$

The following lemmas come from [8, Propositions 3.3-3.5]. 
LemMA 2.2. Assume that $\Omega$ is a bounded domain in $\mathbb{R}^{N}, N \geq 2$, and $\sigma$ satisfies $\left(\mathcal{H}_{\alpha}\right)$. Then the following embeddings hold:

(i) $\mathcal{D}_{0}^{1}(\Omega, \sigma) \hookrightarrow L^{2_{\alpha}^{*}}(\Omega)$ continuously;

(ii) $\mathcal{D}_{0}^{1}(\Omega, \sigma) \hookrightarrow L^{p}(\Omega)$ compactly if $p \in\left[1,2_{\alpha}^{*}\right)$.

Lemma 2.3. Assume that $\Omega$ is an unbounded domain in $\mathbb{R}^{N}, N \geq 2$, and $\sigma$ satisfies $\left(\mathcal{H}_{\alpha, \beta}^{\infty}\right)$. Then the following embeddings hold:

(i) $\mathcal{D}_{0}^{1}(\Omega, \sigma) \hookrightarrow L^{p}(\Omega)$ continuously for every $p \in\left[2_{\beta}^{*}, 2_{\alpha}^{*}\right]$,

(ii) $\mathcal{D}_{0}^{1}(\Omega, \sigma) \hookrightarrow L^{p}(\Omega)$ compactly if $p \in\left(2_{\beta}^{*}, 2_{\alpha}^{*}\right)$.

We now consider the case where $\Omega$ is a bounded domain (the unbounded case is considered similarly with $\left(\mathcal{H}_{\alpha, \beta}^{\infty}\right)$ instead of $\left.\left(\mathcal{H}_{\alpha}\right)\right)$.

We consider the boundary value problem

$$
-\operatorname{div}(\sigma(x) \nabla u)=h \in L^{2}(\Omega), x \in \Omega,\left.\quad u\right|_{\partial \Omega}=0
$$

In order to apply the Friedrichs extension of symmetric operators, we set

$$
X=L^{2}(\Omega), \quad D(\tilde{A})=C_{0}^{\infty}(\Omega), \quad \tilde{A} u=-\operatorname{div}(\sigma(x) \nabla u) .
$$

The problem (2.2) corresponds to the operator equation

$$
\tilde{A} u=h, \quad u \in C_{0}^{\infty}(\Omega), h \in X .
$$

For every $u, v \in C_{0}^{\infty}(\Omega)$, we have

$$
(\tilde{A} u, v)=\int_{\Omega} \sigma(x) \nabla u \nabla v d x=(u, \tilde{A} v) .
$$

It follows from Lemma 2.2 that there exists a constant $C>0$ such that

$$
(\tilde{A} u, u) \geq C\|u\|_{X}^{2}, \quad \text { for any } u \in C_{0}^{\infty}(\Omega)
$$

Hence, $\tilde{A}$ is symmetric and strongly monotone. Applying the Friedrichs extension theorem [25], we find that the energy space $X_{E}$ equals to $\mathcal{D}_{0}^{1}(\Omega, \sigma)$ since $X_{E}$ is the completion of $D(\tilde{A})=C_{0}^{\infty}(\Omega)$ with respect to the scalar product $(u, v)_{\sigma}=$ $\int_{\Omega} \sigma(x) \nabla u \nabla v d x$, and the extensions satisfy

$$
\tilde{A} \subset A \subset A_{E},
$$

where $A_{E}: \mathcal{D}_{0}^{1}(\Omega, \sigma) \rightarrow \mathcal{D}^{-1}(\Omega, \sigma)$ is the energetic extension $\left(\mathcal{D}^{-1}(\Omega, \sigma)\right.$ is the dual space of $\left.\mathcal{D}_{0}^{1}(\Omega, \sigma)\right)$, and $A=-\operatorname{div}(\sigma(x) \nabla)$ is the Friedrichs extension of $\tilde{A}$ with the domain of definition

$$
D(A)=\left\{u \in \mathcal{D}_{0}^{1}(\Omega, \sigma): A u \in X\right\} .
$$

Noticing that $2_{\alpha}^{*}>2$, we have an evolution triple

$$
\mathcal{D}_{0}^{1}(\Omega, \sigma) \hookrightarrow L^{2}(\Omega) \hookrightarrow \mathcal{D}^{-1}(\Omega, \sigma)
$$


with compact and dense embbedings. Hence, there exists a complete orthonormal system of eigenvectors $\left(e_{j}, \lambda_{j}\right)$ such that

$$
\begin{gathered}
\left(e_{j}, e_{k}\right)=\delta_{j k} \quad \text { and } \quad-\operatorname{div}\left(\sigma(x) \nabla e_{j}\right)=\lambda_{j} e_{j}, \quad j, k=1,2, \ldots, \\
0<\lambda_{1} \leq \lambda_{2} \leq \lambda_{3} \leq \ldots, \quad \lambda_{j} \rightarrow+\infty \text { as } j \rightarrow \infty
\end{gathered}
$$

Noting that

$$
\lambda_{1}=\inf \left\{\frac{\|u\|_{\mathcal{D}_{0}^{1}(\Omega, \sigma)}^{2}}{\|u\|_{L^{2}(\Omega)}^{2}}: u \in \mathcal{D}_{0}^{1}(\Omega, \sigma), u \neq 0\right\},
$$

we have

$$
\|u\|_{\mathcal{D}_{0}^{1}(\Omega, \sigma)}^{2} \geq \lambda \lambda_{1}\|u\|_{L^{2}(\Omega)}^{2}, \quad \text { for all } u \in \mathcal{D}_{0}^{1}(\Omega, \sigma) .
$$

2.2. Pullback $\mathcal{D}$-attractors. Let $X$ be a metric space with metric $d$. Denote by $\mathcal{B}(X)$ the set of all bounded subsets of $X$. For $A, B \subset X$, the Hausdorff semi-distance between $A$ and $B$ is defined by

$$
\operatorname{dist}(A, B)=\sup _{x \in A} \inf _{y \in B} d(x, y) .
$$

Let $\{U(t, \tau): t \geq \tau, \tau \in \mathbb{R}\}$ be a process in $X$, i.e. $U(t, \tau): X \rightarrow X$ such that $U(\tau, \tau)=$ $I d$ and $U(t, s) U(s, \tau)=U(t, \tau)$ for all $t \geq s \geq \tau, \tau \in \mathbb{R} .\{U(t, \tau)\}$ is said to be normto-weak continuous if $U(t, \tau) x_{n} \rightarrow U(t, \tau) x$, as $x_{n} \rightarrow x$ in $X$, for all $t \geq \tau, \tau \in$ $\mathbb{R}$. The following result is useful for verifying that a process is norm-to-weak continuous.

Proposition 2.4. [24] Let $X, Y$ be two Banach spaces, $X^{*}, Y^{*}$ be respectively their dual spaces. Assume that $X$ is dense in $Y$, the injection $i: X \rightarrow Y$ is continuous and its adjoint $i^{*}: Y^{*} \rightarrow X^{*}$ is dense, and $\{U(t, \tau)\}$ is a continuous or weak continuous process on $Y$. Then $\{U(t, \tau)\}$ is a norm-to-weak continuous on $X$ if and only if for $t \geq \tau, \tau \in \mathbb{R}$, $U(t, \tau)$ maps a compact set of $X$ to be a bounded set of $X$. $\mathcal{B}(X)$.

Suppose that $\mathcal{D}$ is a non-empty class of parameterised sets $\hat{\mathcal{D}}=\{D(t): t \in \mathbb{R}\} \subset$

Definition 2.1. The process $\{U(t, \tau)\}$ is said to be pullback $\mathcal{D}$-asymptotically compact if for any $t \in \mathbb{R}$, any $\hat{\mathcal{D}} \in \mathcal{D}$, and any sequence $\tau_{n} \rightarrow-\infty$, any sequence $x_{n} \in D\left(\tau_{n}\right)$, the sequence $\left\{U\left(t, \tau_{n}\right) x_{n}\right\}$ is relatively compact in $X$.

Definition 2.2. A process $\{U(t, \tau)\}$ is called pullback $\omega$-D -limit compact if for any $\varepsilon>0$, any $t \in \mathbb{R}$, and $\hat{\mathcal{D}} \in \mathcal{D}$, there exists a $\tau_{0}(\hat{\mathcal{D}}, \varepsilon, t) \leq t$ such that

$$
\alpha\left(\bigcup_{\tau \leq \tau_{0}} U(t, \tau) D(\tau)\right) \leq \varepsilon
$$

where $\alpha$ is the Kuratowski measure of non-compactness of $B \in \mathcal{B}(X)$,

$$
\alpha(B)=\inf \{\delta>0 \mid B \text { has a finite open cover of sets of diameter } \leq \delta\} .
$$


Lemma 2.5. [16] A process $\{U(t, \tau)\}$ is pullback $\mathcal{D}$-asymptotically compact if and only if it is $\omega$-D-limit compact.

Definition 2.3. A family of bounded sets $\hat{\mathcal{B}} \in \mathcal{D}$ is called pullback $\mathcal{D}$-absorbing for the process $\{U(t, \tau)\}$ if for any $t \in \mathbb{R}$, any $\hat{\mathcal{D}} \in \mathcal{D}$, there exists $\tau_{0}=\tau_{0}(\hat{\mathcal{D}}, t) \leq t$ such that

$$
\bigcup_{\tau \leq \tau_{0}} U(t, \tau) D(\tau) \subset B(t) .
$$

Definition 2.4. A family $\hat{\mathcal{A}}=\{A(t): t \in \mathbb{R}\} \subset \mathcal{B}(X)$ is said to be a pullback $\mathcal{D}$ attractor for $\{U(t, \tau)\}$ if

(1) $A(t)$ is compact for all $t \in \mathbb{R}$;

(2) $\hat{\mathcal{A}}$ is invariant, i.e.

$$
U(t, \tau) A(\tau)=A(t), \text { for all } t \geq \tau
$$

(3) $\hat{\mathcal{A}}$ is pullback $\mathcal{D}$-attracting, i.e.

$$
\lim _{\tau \rightarrow-\infty} \operatorname{dist}(U(t, \tau) D(\tau), A(t))=0, \text { for all } \hat{\mathcal{D}} \in \mathcal{D}, \text { and all } t \in \mathbb{R} ;
$$

(4) If $\{C(t): t \in \mathbb{R}\}$ is another family of closed attracting sets then $A(t) \subset C(t)$, for all $t \in \mathbb{R}$.

THEOREM 2.6. [16] Let $\{U(t, \tau)\}$ be a norm-to-weak continuous process such that $\{U(t, \tau)\}$ is pullback $\mathcal{D}$-asymptotically compact. If there exists a family of pullback $\mathcal{D}$ absorbing sets $\hat{\mathcal{B}}=\{B(t): t \in \mathbb{R}\} \in \mathcal{D}$, then $\{U(t, \tau)\}$ has a unique pullback $\mathcal{D}$-attractor $\hat{\mathcal{A}}=\{A(t): t \in \mathbb{R}\}$ and

$$
A(t)=\bigcap_{s \leq t} \overline{\bigcup_{\tau \leq s} U(t, \tau) B(\tau)} .
$$

3. Existence of global solutions. We consider the spaces

$$
\begin{aligned}
V & =L^{2}\left(\tau, T ; \mathcal{D}_{0}^{1}(\Omega, \sigma)\right) \cap L^{p}\left(\tau, T ; L^{p}(\Omega)\right), \\
V^{*} & =L^{2}\left(\tau, T ; \mathcal{D}^{-1}(\Omega, \sigma)\right)+L^{p^{\prime}}\left(\tau, T ; L^{p^{\prime}}(\Omega)\right),
\end{aligned}
$$

where $p^{\prime}$ is the conjugate of $p$. Denote by $|.|_{2},(.,),.\|\|,.((.,)$.$) the norms and scalar$ products in $L^{2}(\Omega)$ and $\mathcal{D}_{0}^{1}(\Omega, \sigma)$ respectively, and $|.|_{p}$ the norm in $L^{p}(\Omega)$.

Definition 3.1. A function $u$ is called a weak solution of $(1.1)$ on $(\tau, T)$ iff

$$
\begin{aligned}
& u \in V, \quad \frac{\partial u}{\partial t} \in V^{*}, \\
& \left.u\right|_{t=\tau}=u_{\tau} \text { a.e. in } \Omega
\end{aligned}
$$

and

$$
\int_{\tau}^{T} \int_{\Omega}\left(\frac{\partial u}{\partial t} \varphi+\sigma \nabla u \nabla \varphi+f(u) \varphi\right)=\int_{\tau}^{T} \int_{\Omega} g \varphi,
$$

for all test functions $\varphi \in V$. 
The following proposition makes the initial condition in problem (1.1) meaningful.

Proposition 3.1. If $u \in V$ and $\frac{\partial u}{\partial t} \in V^{*}$ then $u \in C\left([\tau, T] ; L^{2}(\Omega)\right)$.

Proof. We select a sequence $u_{n} \in C^{1}\left([\tau, T] ; \mathcal{D}_{0}^{1}(\Omega, \sigma) \cap L^{p}(\Omega)\right)$ such that

$$
\left\{\begin{array}{l}
u_{n} \rightarrow u \text { in } V \\
\frac{\partial u_{n}}{\partial t} \rightarrow \frac{\partial u}{\partial t} \text { in } V^{*}
\end{array}\right.
$$

Then, for all $t, t_{0} \in[\tau, T]$, we have

$$
\left|u_{n}(t)-u_{m}(t)\right|_{2}^{2}=\left|u_{n}\left(t_{0}\right)-u_{m}\left(t_{0}\right)\right|_{2}^{2}+2 \int_{t_{0}}^{t}\left\langle u_{n}^{\prime}(s)-u_{m}^{\prime}(s), u_{n}(s)-u_{m}(s)\right\rangle .
$$

We continue, by choosing $t_{0}$ such that

$$
\left|u_{n}\left(t_{0}\right)-u_{m}\left(t_{0}\right)\right|_{2}^{2}=\frac{1}{T-\tau} \int_{\tau}^{T}\left|u_{n}(t)-u_{m}(t)\right|_{2}^{2}
$$

We have

$$
\begin{aligned}
& \int_{\Omega}\left|u_{n}(t)-u_{m}(t)\right|^{2} \\
& \quad=\frac{1}{T-\tau} \int_{\Omega} \int_{\tau}^{T}\left|u_{n}(t)-u_{m}(t)\right|^{2}+2 \int_{\Omega} \int_{t_{0}}^{t}\left(u_{n}^{\prime}(s)-u_{m}^{\prime}(s)\right)\left(u_{n}(s)-u_{m}(s)\right) \\
& \quad \leq \frac{1}{T-\tau} \int_{\Omega} \int_{\tau}^{T}\left|u_{n}(t)-u_{m}(t)\right|^{2}+2\left\|u_{n}^{\prime}-u_{m}^{\prime}\right\|_{V^{*}}\left\|u_{n}-u_{m}\right\|_{V} .
\end{aligned}
$$

Hence, $\left\{u_{n}\right\}$ is a Cauchy sequence in $C\left([\tau, T] ; L^{2}(\Omega)\right)$. Thus the sequence $\left\{u_{n}\right\}$ converges in $C\left([\tau, T] ; L^{2}(\Omega)\right)$ to a function $v \in C\left([\tau, T] ; L^{2}(\Omega)\right)$. Since $u_{n}(t) \longrightarrow u(t) \in L^{2}(\Omega)$ for a.e. $t \in[\tau, T]$, we deduce that $u=v$ a.e. $t \in[\tau, T]$. After redefining on a subset of zero measure, we get $u \in C\left([\tau, T] ; L^{2}(\Omega)\right)$.

THEOREM 3.2. For any $\tau, T \in \mathbb{R}, u_{\tau} \in L^{2}(\Omega)$ given, the problem (1.1) has a unique weak solution $u$ on $(\tau, T)$. Moreover, the following inequality holds

$$
|u(t)|_{2}^{2} \leq e^{-\lambda_{1}(t-\tau)}\left|u_{\tau}\right|_{2}^{2}+\frac{2 k_{1}}{\lambda_{1}}|\Omega|+\frac{e^{-\lambda_{1} t}}{\lambda_{1}} \int_{-\infty}^{t} e^{\lambda_{1} s}|g(s)|_{2}^{2} .
$$

Proof. Consider the approximating solution $u_{n}(t)$ in the form

$$
u_{n}(t)=\sum_{k=1}^{n} u_{n k}(t) e_{k}
$$

where $\left\{e_{j}\right\}_{j=1}^{\infty}$ are eigenvectors of the operator $A:=-\operatorname{div}(\sigma(x) \nabla)$. We get $u_{n}$ from solving the problem

$$
\begin{aligned}
& \left\langle\frac{\partial u_{n}}{\partial t}, e_{k}\right\rangle+\left\langle A u_{n}, e_{k}\right\rangle+\left\langle f\left(u_{n}\right), e_{k}\right\rangle=\left\langle g, e_{k}\right\rangle \\
& \left(u_{n}(\tau), e_{k}\right)=\left(u_{\tau}, e_{k}\right), k=1, \ldots, n
\end{aligned}
$$


Using the Peano theorem, we get the local existence of $u_{n}$. We now establish some a priori estimates for $u_{n}$. We have

$$
\frac{1}{2} \frac{\partial}{\partial t}\left|u_{n}\right|_{2}^{2}+\left\|u_{n}\right\|^{2}+\int_{\Omega} f\left(u_{n}\right) u_{n}=\int_{\Omega} g(t) u_{n} .
$$

Using hypothesis (1.2) and the Cauchy inequality, we get

$$
\frac{1}{2} \frac{\partial}{\partial t}\left|u_{n}\right|_{2}^{2}+\left\|u_{n}\right\|^{2}+C_{1}\left|u_{n}\right|_{p}^{p}-k_{1}|\Omega| \leq \frac{1}{2 \lambda_{1}}|g(t)|_{2}^{2}+\frac{\lambda_{1}}{2}\left|u_{n}\right|_{2}^{2} .
$$

Noting that $\|u\|^{2} \geq \lambda_{1}|u|_{2}^{2}$, we have

$$
\frac{\partial}{\partial t}\left|u_{n}\right|_{2}^{2}+\left\|u_{n}\right\|^{2}+2 C_{1}\left|u_{n}\right|_{p}^{p} \leq \frac{1}{\lambda_{1}}|g(t)|_{2}^{2}+2 k_{1}|\Omega| .
$$

Integrating (3.2) on $[\tau, t], \quad 0<t \leq T$, we have

$$
\left|u_{n}(t)\right|_{2}^{2}+\int_{\tau}^{t}\left\|u_{n}\right\|^{2}+2 C_{1} \int_{\tau}^{t}\left|u_{n}\right|_{p}^{p} \leq\left|u_{n}(\tau)\right|_{2}^{2}+\frac{1}{\lambda_{1}} \int_{\tau}^{t}|g(s)|_{2}^{2}+2 k_{1}|\Omega|(t-\tau) .
$$

The last inequality implies that

$$
\begin{aligned}
& \left\{u_{n}\right\} \text { is bounded in } L^{\infty}\left(\tau, T ; L^{2}(\Omega)\right), \\
& \left\{u_{n}\right\} \text { is bounded in } L^{2}\left(\tau, T ; \mathcal{D}_{0}^{1}(\Omega, \sigma)\right), \\
& \left\{u_{n}\right\} \text { is bounded in } L^{p}\left(\tau, T ; L^{p}(\Omega)\right) .
\end{aligned}
$$

Using hypothesis (1.2), we get the estimates

$$
\int_{\tau}^{T}\left\|f\left(u_{n}\right)\right\|_{L^{p^{\prime}}(\Omega)}^{p^{\prime}} \leq \int_{\tau}^{T} \int_{\Omega} C\left(1+\left|u_{n}\right|^{p-1}\right)^{p^{\prime}} \leq \int_{\tau}^{T} \int_{\Omega} C\left(1+\left|u_{n}\right|^{p}\right) .
$$

Hence $\left\{f\left(u_{n}\right)\right\}$ is bounded in $L^{p^{\prime}}\left(\tau, T ; L^{p^{\prime}}(\Omega)\right)$ and thus

$$
f\left(u_{n}\right) \rightarrow \eta \text { in } L^{p^{\prime}}\left(\tau, T ; L^{p^{\prime}}(\Omega)\right) .
$$

Therefore we have

$$
\begin{aligned}
& u_{n} \rightarrow u \text { in } L^{2}\left(\tau, T ; \mathcal{D}_{0}^{1}(\Omega, \sigma)\right), \\
& f\left(u_{n}\right) \rightarrow \eta \text { in } L^{p^{\prime}}\left(\tau, T ; L^{p^{\prime}}(\Omega)\right), \\
& A u_{n} \rightarrow A u \text { in } L^{2}\left(\tau, T ; \mathcal{D}^{-1}(\Omega, \sigma)\right),
\end{aligned}
$$

up to a subsequence. By rewriting the equation as

$$
\frac{\partial u_{n}}{\partial t}=-A u_{n}-f\left(u_{n}\right)+g
$$

we see that $\left\{\frac{\partial u_{n}}{\partial t}\right\}$ is bounded in $V^{*}$, and therefore in $L^{p^{\prime}}\left(\tau, T ; \mathcal{D}^{-1}(\Omega, \sigma)+L^{p^{\prime}}(\Omega)\right)$. Noting that

$$
\mathcal{D}_{0}^{1}(\Omega, \sigma) \subset \subset L^{2}(\Omega) \subset \mathcal{D}^{-1}(\Omega, \sigma)+L^{p^{\prime}}(\Omega)
$$

is an evolution triplet, applying the Compactness Lemma [18] we can assume that $u_{n} \rightarrow$ $u$ strongly in $L^{2}\left(\tau, T ; L^{2}(\Omega)\right)$. Hence $u_{n} \rightarrow u$ a.e. in $\Omega \times[\tau, T]$. Since $f$ is continuous, 
it follows that $f\left(u_{n}\right) \rightarrow f(u)$ a.e. in $\Omega \times[\tau, T]$. Thanks to (3.7) and Lemma 1.3 in [18, Chapter 1], one has

$$
f\left(u_{n}\right) \rightarrow f(u) \text { in } L^{p^{\prime}}\left(\tau, T ; L^{p^{\prime}}(\Omega)\right)
$$

Thus, from (3.8) we have

$$
u^{\prime}=-A u-f(u)+g \text { in } V^{*}
$$

By Proposition 3.1, we have $u \in C\left([\tau, T] ; L^{2}(\Omega)\right)$. It remains to show that $u(\tau)=u_{\tau}$. Choosing some test function $\varphi \in C^{1}\left([\tau, T] ; \mathcal{D}_{0}^{1}(\Omega, \sigma) \cap L^{p}(\Omega)\right)$ with $\varphi(T)=0$, observe that $\varphi \in V$, so in the 'limiting equation' one can integrate by parts in the $t$ variable to give

$$
\int_{\tau}^{T}-\left(u, \varphi^{\prime}\right)+\int_{\tau}^{T} \int_{\Omega} \sigma(x) \nabla u \nabla \varphi+\int_{\tau}^{T} \int_{\Omega}(f(u)-g) \varphi=(u(\tau), \varphi(\tau)) .
$$

By applying the same procedure to the Galerkin approximations, we get that

$$
\int_{\tau}^{T}-\left(u_{n}, \varphi^{\prime}\right)+\int_{\tau}^{T} \int_{\Omega} \sigma(x) \nabla u_{n} \nabla \varphi+\int_{\tau}^{T} \int_{\Omega}\left(f\left(u_{n}\right)-g\right) \varphi=\left(u_{n}(\tau), \varphi(\tau)\right) .
$$

Taking limits as $n \rightarrow \infty$ we conclude that

$$
\int_{\tau}^{T}-\left(u, \varphi^{\prime}\right)+\int_{\tau}^{T} \int_{\Omega} \sigma(x) \nabla u \nabla \varphi+\int_{\tau}^{T} \int_{\Omega}(f(u)-g) \varphi=\left(u_{\tau}, \varphi(\tau)\right)
$$

since $u_{n}(\tau) \rightarrow u_{\tau}$. Thus, $u(\tau)=u_{\tau}$.

We now prove the uniqueness and the continuous dependence of the solution. Let $u_{1}, u_{2}$ be two solutions of problem (1.1) with the initial data $u_{1}(\tau), u_{2}(\tau)$, respectively. From (1.1), we have

$$
\frac{\partial}{\partial t}\left(u_{1}-u_{2}\right)+\left(A u_{1}-A u_{2}\right)+f\left(u_{1}\right)-f\left(u_{2}\right)=0 .
$$

Since $\left\langle A u_{1}-A u_{2}, u_{1}-u_{2}\right\rangle \geq \lambda_{1}\left|u_{1}-u_{2}\right|_{2}^{2}$ and using (1.3) we deduce that

$$
\begin{aligned}
\frac{1}{2} \frac{\partial}{\partial t}\left|u_{1}-u_{2}\right|_{2}^{2} & \leq \frac{1}{2} \frac{\partial}{\partial t}\left|u_{1}-u_{2}\right|_{2}^{2}+\left\langle A u_{1}-A u_{2}, u_{1}-u_{2}\right\rangle \\
& =-\left\langle f\left(u_{1}\right)-f\left(u_{2}\right), u_{1}-u_{2}\right\rangle \leq l\left|u_{1}-u_{2}\right|_{2}^{2} .
\end{aligned}
$$

Applying the Gronwall lemma, we obtain

$$
\left|u_{1}(t)-u_{2}(t)\right|_{2}^{2} \leq e^{2 l(t-\tau)}\left|u_{1}(\tau)-u_{2}(\tau)\right|_{2}^{2}
$$

This implies the uniqueness (if $u_{1}(\tau)=u_{2}(\tau)$ ) and the continuous dependence of the solution.

Finally, we prove (3.1). Multiplying (1.1) by $u$ and integrating over $\Omega$, we have

$$
\frac{1}{2} \frac{\partial}{\partial t}|u|_{2}^{2}+\|u\|^{2}+\int_{\Omega} f(u) u=\int_{\Omega} g(t) u
$$


Using (1.2) and the Cauchy inequality, we obtain

$$
\frac{\partial}{\partial t}|u|_{2}^{2}+2\|u\|^{2}+2 C_{1}|u|_{p}^{p} \leq 2 k_{1}|\Omega|+\frac{1}{\lambda_{1}}|g(t)|_{2}^{2}+\lambda_{1}|u|_{2}^{2}
$$

Noting that $\|u\|^{2} \geq \lambda_{1}|u|_{2}^{2}$, we have

$$
\frac{\partial}{\partial t}|u|_{2}^{2}+\lambda_{1}|u|_{2}^{2} \leq 2 k_{1}|\Omega|+\frac{1}{\lambda_{1}}|g(t)|_{2}^{2} .
$$

Applying the Gronwall lemma, we get

$$
|u(t)|_{2}^{2} \leq e^{-\lambda_{1}(t-\tau)}\left|u_{\tau}\right|_{2}^{2}+\frac{2 k_{1}}{\lambda_{1}}|\Omega|+\frac{e^{-\lambda_{1} t}}{\lambda_{1}} \int_{-\infty}^{t} e^{\lambda_{1} s}|g(s)|_{2}^{2} .
$$

Hence it follows that the solution $u$ can be extended to $[0,+\infty)$.

4. Existence of pullback $\mathcal{D}$-attractors. Due to the results of Theorem 3.2, we can define a process

$$
U(t, \tau): L^{2}(\Omega) \rightarrow \mathcal{D}_{0}^{1}(\Omega, \sigma) \cap L^{p}(\Omega),
$$

where $U(t, \tau) u_{\tau}$ is the unique solution of (1.1) with the initial data $u_{\tau}$ at time $\tau$.

Lemma 4.1. Assume that $f, g$ satisfy conditions (1.2)-(1.4), and $u(t)$ is the weak solution of problem (1.1). Then the following inequality holds for $t>\tau$ :

$$
\begin{aligned}
|u|_{2}^{2}+\|u\|^{2}+|u|_{p}^{p} \leq c & \left(\left(1+(t-\tau)+\frac{1}{t-\tau}\right) e^{-\lambda_{1}(t-\tau)}\left|u_{\tau}\right|_{2}^{2}+\left(1+\frac{1}{t-\tau}\right)\right. \\
& +\left(1+\frac{1}{t-\tau}\right) e^{-\lambda_{1} t} \int_{-\infty}^{t} e^{\lambda_{1} s}|g(s)|_{2}^{2} \\
& \left.+\left(1+\frac{1}{t-\tau}\right) e^{-\lambda_{1} t} \int_{-\infty}^{t} \int_{-\infty}^{s} e^{\lambda_{1} r}|g(r)|_{2}^{2}\right) .
\end{aligned}
$$

This implies that there exists a family of pullback $\mathcal{D}$-absorbing sets in $\mathcal{D}_{0}^{1}(\Omega, \sigma) \cap L^{p}(\Omega)$ for the process $\{U(t, \tau)\}$.

Proof. Multiplying (3.12) by $e^{\lambda_{1} t}$ and integrating from $\tau$ to $t$, we get

$$
\int_{\tau}^{t} e^{\lambda_{1} s}|u|_{2}^{2} \leq(t-\tau) e^{\lambda_{1} \tau}\left|u_{\tau}\right|_{2}^{2}+\frac{2 k_{1}}{\lambda_{1}^{2}}|\Omega| e^{\lambda_{1} t}+\frac{1}{\lambda_{1}} \int_{-\infty}^{t} \int_{-\infty}^{s} e^{\lambda_{1} r}|g(r)|^{2} .
$$

Using (3.10) and the fact that $\|u\|^{2} \geq \lambda_{1}|u|_{2}^{2}$, we have

$$
\frac{\partial}{\partial t}|u|_{2}^{2}+\|u\|^{2}+2 C_{1}|u|_{p}^{p} \leq 2 k_{1}|\Omega|+\frac{1}{\lambda_{1}}|g(t)|_{2}^{2}
$$

thus

$$
\frac{\partial}{\partial t}\left(e^{\lambda_{1} t}|u|_{2}^{2}\right)+e^{\lambda_{1} t}\left(\|u\|^{2}+2 C_{1}|u|_{p}^{p}\right) \leq \lambda_{1} e^{\lambda_{1} t}|u|_{2}^{2}+2 k_{1}|\Omega| e^{\lambda_{1} t}+\frac{e^{\lambda_{1} t}}{\lambda_{1}}|g(t)|_{2}^{2} .
$$


Integrating from $\tau$ to $t$ and using (4.2), we have

$$
\begin{aligned}
\int_{\tau}^{t} e^{\lambda_{1} s}\left(\|u\|^{2}+2 C_{1}|u|_{p}^{p}\right) \leq & \left(1+\lambda_{1}(t-\tau)\right) e^{\lambda_{1} \tau}\left|u_{\tau}\right|_{2}^{2}+\frac{4 k_{1}}{\lambda_{1}}|\Omega| e^{\lambda_{1} t} \\
& +\frac{1}{\lambda_{1}} \int_{-\infty}^{t} e^{\lambda_{1} s}|g(s)|_{2}^{2}+\int_{-\infty}^{t} \int_{-\infty}^{s} e^{\lambda_{1} r}|g(r)|^{2} .
\end{aligned}
$$

Combining (4.2) and (4.5), we get

$$
\begin{aligned}
\int_{\tau}^{t} e^{\lambda_{1} s}\left(\|u\|^{2}+2 C_{1}|u|_{p}^{p}+|u|_{2}^{2}\right) \leq & \left(1+\left(\lambda_{1}+1\right)(t-\tau)\right) e^{\lambda_{1} \tau}\left|u_{\tau}\right|_{2}^{2} \\
& +\frac{2 k_{1}\left(2 \lambda_{1}+1\right)}{\lambda_{1}^{2}}|\Omega| e^{\lambda_{1} t}+\frac{1}{\lambda_{1}} \int_{-\infty}^{t} e^{\lambda_{1} s}|g(s)|_{2}^{2} \\
& +\left(1+\frac{1}{\lambda_{1}}\right) \int_{-\infty}^{t} \int_{-\infty}^{s} e^{\lambda_{1} r}|g(r)|_{2}^{2}
\end{aligned}
$$

From (1.2) we deduce that

$$
\tilde{C}_{1}|s|^{p}-\tilde{C}_{2} \leq F(s) \leq \tilde{C}_{3}|s|^{p}+\tilde{C}_{4},
$$

where $F(s)=\int_{0}^{s} f(\tau) d \tau$. Combining (4.3) and (4.7), we get

$$
\frac{\partial}{\partial t}|u|_{2}^{2}+\|u\|^{2}+C_{5} \int_{\Omega} F(u) \leq \frac{1}{\lambda_{1}}|g(t)|_{2}^{2}+C_{7} .
$$

Mutiply (1.1) by $u_{t}$ and integrating over $\Omega$, we have

$$
\left|u_{t}\right|_{2}^{2}+\frac{1}{2} \frac{\partial}{\partial t}\left(\|u\|^{2}+2 \int_{\Omega} F(u)\right)=\int_{\Omega} g(t) u_{t} \leq \frac{1}{2}|g(t)|_{2}^{2}+\frac{1}{2}\left|u_{t}\right|_{2}^{2},
$$

thus

$$
\frac{\partial}{\partial t}\left(\|u\|^{2}+2 \int_{\Omega} F(u)\right) \leq|g(t)|_{2}^{2} .
$$

Using (4.8), (4.9) and (2.3), we have

$$
\frac{\partial}{\partial t} G(u)+C_{8} G(u) \leq C_{9}|g(t)|_{2}^{2}+C_{7},
$$

where $G(u)=|u|_{2}^{2}+\|u\|^{2}+2 \int_{\Omega} F(u)$, which implies

$$
\frac{\partial}{\partial t}\left((t-\tau) e^{\lambda_{1} t} G(u)\right) \leq\left(1+\left(\lambda_{1}-C_{8}\right)(t-\tau)\right) G(u) e^{\lambda_{1} t}+\left(C_{7}+C_{9}|g(t)|_{2}^{2}\right)(t-\tau) e^{\lambda_{1} t} .
$$

Integrating from $\tau$ to $t$, we get

$$
(t-\tau) G(u) \leq\left(1+C_{11}(t-\tau)\right) \int_{\tau}^{t} G(u) e^{\lambda_{1} s}+C_{10}(t-\tau) e^{\lambda_{1} t}+C_{9}(t-\tau) \int_{\tau}^{t} e^{\lambda_{1} s}|g(s)|_{2}^{2} .
$$

Using (4.6) we get the desired inequality (4.1).

Let $\mathcal{R}$ be the set of all functions $r: \mathbb{R} \rightarrow(0,+\infty)$ such that $\lim _{t \rightarrow-\infty} t e^{\lambda_{1} t} r^{2}(t)=0$ and denote by $\mathcal{D}$ the class of all families $\hat{\mathcal{D}}=\{D(t): t \in \mathbb{R}\} \subset \mathcal{B}\left(L^{2}(\Omega)\right)$ such that 
$D(t) \subset \bar{B}(r(t))$ for some $r(t) \in \mathcal{R}$, where $\bar{B}(r(t))$ denotes the closed ball in $L^{2}(\Omega)$ with radius $r(t)$. Let

$$
r_{0}(t)=2 c\left(1+e^{-\lambda_{1} t} \int_{-\infty}^{t} e^{\lambda_{1} s}|g(s)|_{2}^{2}+e^{-\lambda_{1} t} \int_{-\infty}^{t} \int_{-\infty}^{s} e^{\lambda_{1} r}|g(r)|_{2}^{2}\right) .
$$

Condition (1.4) implies the existence of the following integrals

$$
\int_{-\infty}^{t} e^{\lambda_{1} s}|g(s)|_{2}^{2}<\infty \quad \text { and } \int_{-\infty}^{t} \int_{-\infty}^{s} e^{\lambda_{1} r}|g(r)|_{2}^{2}<\infty \quad \text { for all } t \in \mathbb{R} .
$$

We denote by $\bar{B}_{0}\left(r_{0}(t)\right)$ the closed ball in $\mathcal{D}_{0}^{1}(\Omega, \sigma) \cap L^{p}(\Omega)$ centred at 0 with radius $r_{0}(t)$. Obviously for any $\hat{\mathcal{D}} \in \mathcal{D}$ and any $t \in \mathbb{R}$, by (4.1) there exists $\tau_{0}=\tau_{0}(\hat{\mathcal{D}}, t) \leq t$ such that

$$
|u|_{2}^{2}+\|u\|^{2}+|u|_{p}^{p} \leq r_{0}(t) \text { for all } \tau \leq \tau_{0},
$$

i.e. $\hat{\mathcal{B}}=\left\{\bar{B}_{0}\left(r_{0}(t)\right): t \in \mathbb{R}\right\}$ is a family of bounded pullback $\mathcal{D}$-absorbing sets in $\mathcal{D}_{0}^{1}(\Omega, \sigma) \cap L^{p}(\Omega)$.

From the above lemma we deduce that the process $\{U(t, \tau)\}$ maps a compact set of $\mathcal{D}_{0}^{1}(\Omega, \sigma) \cap L^{p}(\Omega)$ to be a bounded set of $\mathcal{D}_{0}^{1}(\Omega, \sigma) \cap L^{p}(\Omega)$, and thus by Proposition 2.4 , the process $\{U(t, \tau)\}$ is norm-to-weak continuous in $\mathcal{D}_{0}^{1}(\Omega, \sigma) \cap L^{p}(\Omega)$. Since $\{U(t, \tau)\}$ has a family of pullback $\mathcal{D}$-absorbing sets in $\mathcal{D}_{0}^{1}(\Omega, \sigma) \cap L^{p}(\Omega)$, in order to prove the existence of pullback $\mathcal{D}$-attractors, we only need to check that $\{U(t, \tau)\}$ is pullback $\mathcal{D}$-asymptotically compact.

4.1. Pullback $\mathcal{D}$-attractor in $L^{2}(\Omega)$. Since $\mathcal{D}_{0}^{1}(\Omega, \sigma) \hookrightarrow L^{2}(\Omega)$ compactly, we immediately get the following result.

THEOREM 4.2. Assume that $f, g$ satisfy conditions (1.2)-(1.4). Then the process corresponding to problem $(1.1)$ has a pullback $\mathcal{D}$-attractor in $L^{2}(\Omega)$.

4.2. Pullback $\mathcal{D}$-attractor in $L^{p}(\Omega)$. To prove that the $\{U(t, \tau)\}$ is pullback $\mathcal{D}$ asymptotically compact in $L^{p}(\Omega)$, we need the following lemma.

LEMMA 4.3. Let $\{U(t, \tau)\}$ be a norm-to-weak continuous process in $L^{2}(\Omega)$ and $L^{p}(\Omega)$, and $\{U(t, \tau)\}$ satisfy the following two conditions:

(i) $\{U(t, \tau)\}$ is pullback $\mathcal{D}$-asymptotically compact in $L^{2}(\Omega)$;

(ii) for any $\varepsilon>0, \hat{\mathcal{B}} \in \mathcal{D}$, there exist constants $M(\varepsilon, \hat{\mathcal{B}})$ and $\tau_{0}(\varepsilon, \hat{\mathcal{B}}) \leq t$ such that:

$$
\left(\int_{\Omega\left(\left|U(t, \tau) u_{\tau}\right| \geq M\right)}\left|U(t, \tau) u_{\tau}\right|^{p}\right)^{\frac{1}{p}}<\varepsilon, \quad \text { for any } u_{\tau} \in B(\tau), \text { and } \tau \leq \tau_{0} .
$$

Then $\{U(t, \tau)\}$ is pullback $\mathcal{D}$-asymptotically compact in $L^{p}(\Omega)$.

Proof. For any fixed $\varepsilon>0$, and $\hat{\mathcal{B}} \in \mathcal{D}$, it follows from condition (i) and Lemma 2.5 that there exists $\tau_{1}=\tau_{1}(\hat{\mathcal{B}}, \varepsilon) \leq \tau_{0}$ such that

$$
\alpha\left(\bigcup_{\tau \leq \tau_{1}} U(t, \tau) B(\tau)\right) \leq(3 M)^{\frac{2-p}{2}}\left(\frac{\varepsilon}{2}\right)^{\frac{p}{2}} \text { in } L^{2}(\Omega)
$$


i.e. $\bigcup_{\tau \leq \tau_{1}} U(t, \tau) B(\tau)$ has a finite $(3 M)^{\frac{2-p}{2}}\left(\frac{\varepsilon}{2}\right)^{\frac{p}{2}}$-net in $L^{2}(\Omega)$. By Lemma 5.3 in [24], $\bigcup_{\tau \leq \tau_{1}} U(t, \tau) B(\tau)$ has a finite $\varepsilon$-net in $L^{p}(\Omega)$. By the definition of the measure of non-compactness, we obtain

$$
\alpha\left(\bigcup_{\tau \leq \tau_{1}} U(t, \tau) B(\tau)\right) \leq \varepsilon \text { in } L^{p}(\Omega),
$$

i.e. $\{U(t, \tau)\}$ is pullback $\omega$-D-limit compact in $L^{p}(\Omega)$. Applying the results of Lemma 2.5 once again, we deduce that $\{U(t, \tau)\}$ is pullback $\mathcal{D}$-asymptotically compact in $L^{p}(\Omega)$.

THEOREM 4.4. Assume that $f, g$ satisfy conditions (1.2)-(1.4). Then the process corresponding to problem (1.1) has a pullback $\mathcal{D}$-attractor in $L^{p}(\Omega)$.

Proof. It is sufficient to show that the process $\{U(t, \tau)\}$ satisfies the condition (ii) in Lemma 4.3. In fact, take $M$ large enough such that $C_{1}|u|^{p-1} \leq f(u)$ in

$$
\Omega_{1}=\Omega(u(t) \geq M)=\{x \in \Omega: u(x, t) \geq M\},
$$

and denote

$$
(u-M)^{+}= \begin{cases}u-M, & u \geq M \\ 0, & u<M\end{cases}
$$

In $\Omega_{1}$ we see that

$$
\begin{aligned}
g(t)\left((u-M)^{+}\right)^{p-1} & \leq \frac{C_{1}}{2}\left((u-M)^{+}\right)^{2 p-2}+\frac{1}{2 C_{1}}|g(t)|^{2} \\
& \leq \frac{C_{1}}{2}\left((u-M)^{+}\right)^{p-1}|u|^{p-1}+\frac{1}{2 C_{1}}|g(t)|^{2},
\end{aligned}
$$

and

$$
\begin{aligned}
f(u)\left((u-M)^{+}\right)^{p-1} & \geq C_{1}|u|^{p-1}\left((u-M)^{+}\right)^{p-1} \\
& \geq \frac{C_{1}}{2}\left((u-M)^{+}\right)^{p-1}|u|^{p-1}+\frac{C_{1} M^{p-2}}{2}\left((u-M)^{+}\right)^{p} .
\end{aligned}
$$

Multiplying (1.1) by $\left|(u-M)^{+}\right|^{p-1}$ and using (4.10), (4.11), we deduce that

$$
\begin{aligned}
& \frac{1}{p} \frac{\partial}{\partial t}\left|(u-M)^{+}\right|_{p}^{p}+(p-1) \int_{\Omega_{1}} \sigma(x)\left|\nabla(u-M)^{+}\right|^{2}\left|(u-M)^{+}\right|^{p-2} \\
& \quad+C_{1} M^{p-2} \int_{\Omega_{1}}\left|(u-M)^{+}\right|^{p} \\
& \quad \leq \int_{\Omega_{1}} \frac{1}{C_{1}}|g(t)|^{2}
\end{aligned}
$$

Therefore

$$
\frac{\partial}{\partial t}\left|(u-M)^{+}\right|_{p}^{p}+C M^{p-2}\left|(u-M)^{+}\right|_{p}^{p} \leq C|g(t)|_{2}^{2},
$$


which implies that

$$
\frac{\partial}{\partial t}(t-\tau) e^{C M^{p-2} t}\left|(u-M)^{+}\right|_{p}^{p} \leq e^{C M^{p-2} t}\left|(u-M)^{+}\right|_{p}^{p}+C(t-\tau) e^{C M^{p-2} t}|g(t)|_{2}^{2}
$$

Integrating (4.12) from $\tau$ to $t$, we get

$$
\begin{aligned}
(t-\tau) e^{C M^{p-2} t}\left|(u-M)^{+}\right|_{p}^{p} & \leq \int_{\tau}^{t} e^{C M^{p-2} t}\left|(u-M)^{+}\right|_{p}^{p}+C(t-\tau) \int_{\tau}^{t} e^{C M^{p-2} t}|g(t)|_{2}^{2} \\
& \leq e^{\left(C M^{p-2}-\lambda_{1}\right) t} \int_{\tau}^{t} e^{\lambda_{1} s}|u|_{p}^{p}+\frac{C(t-\tau) e^{\left(C M^{p-2}-\gamma\right) t}}{C M^{p-2}-\gamma}
\end{aligned}
$$

and then

$$
\left|(u-M)^{+}\right|_{p}^{p} \leq \frac{1}{t-\tau} e^{-\lambda_{1} t} \int_{\tau}^{t} e^{\lambda_{1} s}|u|_{p}^{p}+\frac{C e^{-\gamma t}}{C M^{p-2}-\gamma} .
$$

By (4.13) and (4.6), we obtain

$$
\begin{aligned}
&\left|(u-M)^{+}\right|_{p}^{p} \leq C( \\
&\left(1+\frac{1}{t-\tau}\right) e^{-\lambda_{1}(t-\tau)}\left|u_{\tau}\right|_{2}^{2}+\frac{1}{t-\tau}+\frac{e^{-\lambda_{1} t}}{t-\tau} \int_{-\infty}^{t} e^{\lambda_{1} s}|g(s)|_{2}^{2} \\
&\left.+\frac{e^{-\lambda_{1} t}}{t-\tau} \int_{-\infty}^{t} \int_{-\infty}^{s} e^{\lambda_{1} r}|g(r)|_{2}^{2}\right)+\frac{C e^{-\gamma t}}{C M^{p-2}-\gamma}
\end{aligned}
$$

Hence, for any $\varepsilon>0$, there exist $M_{1}>0$ and $\tau_{1}<t$ such that for any $\tau<\tau_{1}$ and any $M \geq M_{1}$, we have

$$
\int_{\Omega(u(t) \geq M)}\left|(u-M)^{+}\right|^{p} \leq \varepsilon .
$$

Repeating the same step above, by just taking $(u+M)^{-}$instead of $(u-M)^{+}$, we deduce that there exist $M_{2}>0$ and $\tau_{2}<t$ such that for any $\tau<\tau_{2}$ and any $M \geq M_{2}$,

$$
\int_{\Omega(u(t) \leq-M)}\left|(u+M)^{-}\right|^{p} \leq \varepsilon
$$

where

$$
(u+M)^{-}= \begin{cases}u+M, & u \leq-M \\ 0, & u \geq-M\end{cases}
$$

Let $M_{0}=\max \left\{M_{1}, M_{2}\right\}$ and $\tau_{0}=\min \left\{\tau_{1}, \tau_{2}\right\}$, we obtain

$$
\int_{\Omega(|u| \geq M)}(|u|-M)^{p} \leq \varepsilon \quad \text { for } \tau \leq \tau_{0} \text { and } M \geq M_{0} .
$$


Using (4.14) and (4.15), we have

$$
\begin{aligned}
\int_{\Omega(|u| \geq 2 M)}|u|^{p} & =\int_{\Omega(|u| \geq 2 M)}((|u|-M)+M)^{p} \\
& \leq 2^{p-1}\left(\int_{\Omega(|u| \geq 2 M)}(|u|-M)^{p}+\int_{\Omega(|u| \geq 2 M)} M^{p}\right) \\
& \leq 2^{p-1}\left(\int_{\Omega(|u| \geq M)}(|u|-M)^{p}+\int_{\Omega(|u| \geq M)}(|u|-M)^{p}\right) \\
& \leq 2^{p} \varepsilon .
\end{aligned}
$$

This completes the proof.

\subsection{Pullback $\mathcal{D}$-attractor in $\mathcal{D}_{0}^{1}(\Omega, \sigma) \cap L^{p}(\Omega)$.}

THEOREM 4.5. Assume that $f, g$ satisfy conditions (1.2)-(1.4). Then the process corresponding to problem $(1.1)$ has a pullback $\mathcal{D}$-attractor in $\mathcal{D}_{0}^{1}(\Omega, \sigma) \cap L^{p}(\Omega)$.

Proof. By Lemma 4.1, $\{U(t, \tau)\}$ has a family of bounded pullback $\mathcal{D}$-absorbing sets in $\mathcal{D}_{0}^{1}(\Omega, \sigma) \cap L^{p}(\Omega)$. It remains to show that $\{U(t, \tau)\}$ is pullback $\mathcal{D}$-asymptotically compact in $\mathcal{D}_{0}^{1}(\Omega, \sigma) \cap L^{p}(\Omega)$, i.e. for any $t \in \mathbb{R}$, any $\hat{\mathcal{B}} \in \mathcal{D}$, any sequence $\tau_{n} \rightarrow-\infty$, and any sequence $u_{\tau_{n}} \in B\left(\tau_{n}\right)$, the sequence $\left\{U\left(t, \tau_{n}\right) u_{\tau_{n}}\right\}$ is precompact in $\mathcal{D}_{0}^{1}(\Omega, \sigma) \cap$ $L^{p}(\Omega)$. Thanks to Theorem 4.4 , we need only to show that the sequence $\left\{U\left(t, \tau_{n}\right) u_{\tau_{n}}\right\}$ is precompact in $\mathcal{D}_{0}^{1}(\Omega, \sigma)$.

We consider the sequence $u_{n}(t)=U\left(t, \tau_{n}\right) u_{\tau_{n}}$, by Theorems 4.2 and 4.4 , we can assume that $\left\{u_{n}(t)\right\}$ is a Cauchy sequence both in $L^{2}(\Omega)$ and $L^{p}(\Omega)$. We have

$$
\begin{aligned}
\left\|u_{n}(t)-u_{m}(t)\right\| & =\left\langle A u_{n}(t)-A u_{m}(t), u_{n}(t)-u_{m}(t)\right\rangle \\
& =\left\langle\frac{d u_{n}}{d t}(t)-\frac{d u_{m}}{d t}(t), u_{n}(t)-u_{m}(t)\right\rangle+\left\langle f\left(u_{n}(t)\right)-f\left(u_{m}(t)\right), u_{n}(t)-u_{m}(t)\right\rangle \\
& \leq \frac{1}{2} \frac{\partial}{\partial t}\left|u_{n}(t)-u_{m}(t)\right|_{2}^{2}+\left|f\left(u_{n}(t)\right)-f\left(u_{m}(t)\right)\right|_{p^{\prime}}\left|u_{n}(t)-u_{m}(t)\right|_{p} .
\end{aligned}
$$

From (3.9) and since $\left\{u_{n}(t)\right\}$ is a Cauchy sequence in $L^{2}(\Omega)$, we get

$$
\frac{\partial}{\partial t}\left|u_{n}(t)-u_{m}(t)\right|_{2}^{2} \rightarrow 0, \quad \text { as } n, m \rightarrow \infty
$$

Using (1.2), we have

$$
\left\|f\left(u_{n}(t)\right)\right\|_{L^{p^{\prime}}(\Omega)}^{p^{\prime}} \leq \int_{\Omega} C\left(1+\left|u_{n}(t)\right|^{p-1}\right)^{p^{\prime}} \leq \int_{\Omega} C\left(1+\left|u_{n}(t)\right|^{p}\right),
$$

thus $\left\{f\left(u_{n}(t)\right)\right\}$ is bounded in $L^{p^{\prime}}(\Omega)$ because $\left\{u_{n}(t)\right\}$ is bounded in $L^{p}(\Omega)$. It follows from here and (4.17), (4.18) that

$$
\left\|u_{n}(t)-u_{m}(t)\right\| \rightarrow 0, \quad \text { as } m, n \rightarrow \infty .
$$

The proof is complete.

5. Some remarks on the case of an unbounded domain. In this section, we comment the case of an unbounded domain $\Omega \subset \mathbb{R}^{N}, N \geq 2$. We assume that the weight function 
$\sigma(x)$ satisfies the condition $\left(\mathcal{H}_{\alpha, \beta}^{\infty}\right)$. It is crucial to note that the embedding $\mathcal{D}_{0}^{1}(\Omega, \sigma) \subset$ $L^{2}(\Omega)$ is compact either condition $\left(\mathcal{H}_{\alpha}\right)$ or $\left(\mathcal{H}_{\alpha, \beta}^{\infty}\right)$ holds. Observe that $\beta>2$ implies $2_{\beta}^{*}:=\frac{2 N}{N-2+\beta}<2$, i.e. $2 \in\left(2_{\beta}^{*}, 2_{\alpha}^{*}\right)$. In the unbounded case we need $\sigma$ to growth faster than quadratically at infinity, to ensure the compactness of the above embedding. Note also that in the case of an unbounded domain, (2.1) does not hold in general, if $\beta \leq 2$. We refer to the examples of [1].

When $\left(\mathcal{H}_{\alpha, \beta}^{\infty}\right)$ is satisfied, the operator $A=-\operatorname{div}(\sigma(x) \nabla)$ has the same properties as in the case of a bounded domain. Therefore, we may apply the methods used for a bounded domain to this case with some small changes in the conditions of $f$. More precisely, we assume that $f: \Omega \times \mathbb{R} \rightarrow \mathbb{R}$ is a Caratheodory function such that

$$
\begin{aligned}
|f(x, u)| & \leq C_{1}|u|^{p-1}+h_{1}(x), \\
f(x, u) u & \geq C_{2}|u|^{p}-h_{2}(x), \\
(f(x, u)-f(x, v))(u-v) & \geq-l|u-v|^{2}, \\
F(x, u) & \geq C_{3}|u|^{p}+h_{3}(x),
\end{aligned}
$$

where $F(x, u)=\int_{0}^{u} f(x, s) d s ; C_{1}, C_{2}, C_{3}, l>0 ; h_{2}(x) \in L^{1}(\Omega)$ and $h_{1}, h_{3} \in L^{p^{\prime}}(\Omega)$ are non-negative real-valued functions, and the external force $g$ satisfies

$$
g \in L_{l o c}^{2}\left(\mathbb{R} ; L^{2}(\Omega)\right) \text {, and }\|g(t)\|_{L^{2}(\Omega)}^{2} \leq M e^{\gamma|t|}, \gamma<\lambda_{1} .
$$

We may now repeat the arguments used in Sections 3 and 4 to obtain

THEOREM 5.1. Under conditions $\left(\mathcal{H}_{\alpha, \beta}^{\infty}\right)$ and (5.1)-(5.5), problem (1.1) defines a process having a pullback $\mathcal{D}$-attractor in $\left.\mathcal{D}_{0}^{1}(\Omega, \sigma) \cap L^{p}(\Omega)\right)$.

ACKNOWLEDGEMENT. This work was supported by Vietnam's National Foundation for Science and Technology Development (NAFOSTED).

\section{REFERENCES}

1. B. Abdelaoui and I. Peral, On quasilinear elliptic equations related to some CaffarelliKohn-Nirenberg inequalities, Commun. Pure Appl. Anal. 2 (2003), 539-566.

2. C. T. Anh and P. Q. Hung, Global existence and long-time behavior of solutions to a class of degenerate parabolic equations, Ann. Pol. Math. 93(3) (2008), 217-230.

3. C. T. Anh and T. D. Ke, Long-time behavior for quasilinear parabolic equations involving weighted $p$-Laplacian operators, Nonlinear Anal. 71 (2009), 4415-4422.

4. C. T. Anh, N. M. Chuong and T. D. Ke, Global attractor for the $m$-semiflow generated by a quasiliner degenerate parabolic equation, J. Math. Anal. Appl. 363 (2010), 444-453.

5. J. M. Ball, On the asymptotic behavior of generalized processes with applications to nonlinear evolution equations, J. Differ. Equ. 27 (1978), 224-265.

6. J. M. Ball, Continuity properties and global attractor of generalized semiflows and the Navier-Stokes equations, J. Nonlinear Sci. 7 (1997), 475-502.

7. J. M. Ball, Global attractor for damped semilinear wave equations, Discrete Contin. Dyn. Syst. 10 (2004), 31-52.

8. P. Caldiroli and R. Musina, On a variational degenerate elliptic problem, Nonlinear Diff. Equ. Appl. 7 (2000), 187-199.

9. T. Caraballo, G. Lukasiewicz and J. Real, Pullback attractors for asymptotically compact non-autonomous dynamical systems, Nonlinear Anal. 64 (2006), $484-498$.

10. T. Caraballo, P. Marin-Rubio and J. C. Robinson, A comparision between to theories for multi-valued semiflows and their asymptotic behaviour, Set-Valued Anal. 11 (2003), 297-322. 
11. V. V. Chepyzhov and M. I. Vishik, Evolution equations and their trajectory attractor, J. Math. Pures App. 76 (1997), 913-964.

12. V. V. Chepyzhov and M. I. Vishik, Attractors for equations of mathematical physics, American Mathematical Society Colloquium, vol. 49 (American Mathematical Society, Providence, RI, 2002).

13. R. Dautray, J. L. Lions, Mathematical analysis and numerical methods for science and technology, Vol. I: Physical origins and classical methods (Springer-Verlag, Berlin, 1985).

14. N. I. Karachalios and N. B. Zographopoulos, Convergence towards attractors for a degenerate Ginzburg-Landau equation, Z. Angew. Math. Phys. 56 (2005), 11-30.

15. N. I. Karachalios and N. B. Zographopoulos, On the dynamics of a degenerate parabolic equation: Global bifurcation of stationary states and convergence, Calc. Var. Partial Differ. Equ. 25(3) (2006), 361-393.

16. Y. Li and C. K. Zhong, Pullback attractors for the norm-to-weak continuous process and application to the nonautonomous reaction-diffusion equations, Appl. Math. Comput. 190 (2007), 1020-1029.

17. Y. Li, S. Wang and H. Wu, Pullback attractors for non-autonomous reaction-diffusion equations in $L^{p}$, Appl. Math. Comput. 207 (2009), 373-379.

18. J.-L. Lions, Quelques Méthodes de Résolution des Problèmes aux Limites Non Linéaires (Dunod, Paris, 1969).

19. S. S. Lu, H. Q. Wu and C. K. Zhong, Attractors for nonautonomous $2 \mathrm{D}$ Navier-Stokes equations with normal external force, Discrete Contin. Dyn. Syst. 23 (2005), 701-719.

20. Q. F. Ma, S. H. Wang and C. K. Zhong, Necessary and sufficient conditions for the existence of global attractor for semigroups and applications, Indian Univ. Math. J. 51(6) (2002), $1541-1559$.

21. V. S. Melnik, J. Valero, On attractors of multi-valued semiflows and differential inclusions, Set-Valued Anal. 6 (1998), 83-111.

22. H. T. Song and H. Q. Wu, Pullback attractors of nonautonomous reaction diffusion equations, J. Math. Anal. Appl. 325 (2007), 1200-1215.

23. M. Vishik, Asymptotic behaviour of solutions of evolutionary equations (Cambridge University Press, Cambridge, UK, 1992).

24. C. K. Zhong, M. H. Yang and C. Y. Sun, The existence of global attractors for the normto-weak continuous semigoup and application to the nonlinear reaction-diffusion equations, J. Differ. Equ. 15 (2006), 367-399.

25. E. Zeidler, Nonlinear functional analysis and its applications, vol. II (Springer-Verlag, New York, 1990). 